\title{
Presentation and pattern of childhood renal diseases in Gusau, North-Western Nigeria
}

\author{
B I Garba, ${ }^{1}$ MBBS, FMCPaed, Dip Allerg, MSc (Med); A S Muhammad, ${ }^{2}$ MBBS, FMCP, MSc (Med); \\ A B Obasi, ${ }^{1} \mathrm{MBBS} ;$ A O Adeniji, ${ }^{1} \mathrm{MBBS}$ \\ ${ }^{1}$ Department of Paediatrics, Ahmad Sani Yariman Bakura Specialist Hospital, Gusau, Zamfara State, Nigeria \\ ${ }^{2}$ Department of Medicine, Ahmad Sani Yariman Bakura Specialist Hospital, Gusau, Zamfara State, Nigeria
}

Corresponding author: B I Garba (bgilah@yahoo.com)

\begin{abstract}
Background. Studies from different parts of Nigeria and the world have reported variable patterns of renal diseases in childhood. There is a paucity of data to guide resource allocation in Zamfara, Nigeria, despite the rising incidence of kidney diseases in children in Nigeria, and globally.

Objectives. To determine the prevalence, presentation, pattern, and outcomes of renal diseases among hospitalised children in Gusau, Zamfara State, Nigeria.

Methods. A retrospective study was conducted of children aged 1 month to 14 years, who were admitted to the paediatric wards of our hospital over a period of 30 months (October 2013 to March 2016). Relevant information was retrieved from the patients' medical records and data were analysed accordingly.

Results. A total of 2658 children were admitted, of which 3.2\% ( $n=84)$ had renal diseases; however, only 70 folders were utilised for the study. The male:female ratio was $1.19: 1$. Fever (63\%), reduction in urine volume/frequency (46\%), body swelling (43\%) and abdominal pain $(40 \%)$ were the most common symptoms. Hypertension $(33 \%)$ and heart failure $(17 \%)$ were common findings. Urinary tract infection (UTI) (34\%), acute glomerulonephritis (AGN) (24\%) and acute kidney injury (AKI) (20\%) were the the most common diagnoses. Most of the children were discharged with good renal function and mortality was low (10\%).

Conclusions. The prevalence of renal disease in our setting was low, with males predominating. UTI was the most common cause of renal disease, requiring hospitalisation in Gusau, while congenital anomalies and malignancies were rare. These data could be utilised by researchers and stakeholders in resource-poor settings like ours to plan for preventive nephrology as UTI, AGN and AKI are largely preventable.,
\end{abstract}

S Afr J Child Health 2017;11(2):96-98. DOI:10.7196/SAJCH.2017.v11i2.1222

Childhood renal diseases are common causes of morbidity and mortality; studies from different parts of Nigeria and the world have reported variable patterns of renal diseases in childhood. Abdurrahman et al. ${ }^{[1]}$ in Zaria, Ocheke et al. ${ }^{[2]}$ in Jos, and Adedoyin et $a l .{ }^{[3]}$ in Ilorin carried out evaluations of childhood renal diseases prevalent in Northern Nigeria. Morbidities of importance noted by these researchers included urinary tract infection (UTI), acute glomerulonephritis (AGN), acute kidney injury (AKI) from varying causes, nephrotic syndrome, congenital urinary tract obstructions and malignancies, with varying rates.

A recent editorial on childhood kidney diseases in developing countries highlights flawed epidemiological data on renal diseases, especially in Africa, with a paucity of data to guide for resource allocation, despite the rising incidence of kidney diseases in children. ${ }^{[4]}$ Regular audits in different hospitals could provide data that would guide healthcare stakeholders in planning for preventive nephrology, which is necessary to reduce the morbidity and mortality from renal diseases.

To our knowledge, the burden of childhood renal diseases in Gusau, Zamfara State, North-Western Nigeria, has not been investigated. In this study, we aimed to determine the prevalence, presentation, pattern and outcome of renal diseases among hospitalised children in Gusau, Zamfara State, Nigeria.

\section{Methods}

This was a retrospective study of children aged 1 month to 14 years, who were admitted to the paediatric wards (emergency paediatric unit and paediatric medical ward) of Ahmad Sani Yariman Bakura
Specialist Hospital (ASYBSH), Gusau, Nigeria, over a period of 30 months between October 2013 and March 2016. We reviewed the records of all patients with a diagnosis of renal disease. Incomplete records and readmissions were excluded from the study. Information about age, gender, history, examination, urine microscopy, and urinalysis was carefully recorded. Depending on the provisional diagnoses, further investigations were carried out, including: serum urea and creatinine; serum protein; lipids; throat swab microscopy, culture and sensitivity; abdominal ultrasound; other imaging studies and renal biopsy. Haemodialysis is available to children aged $\geq 10$ years (depending on their body size) at ASYBSH; however, there are no facilities for peritoneal dialysis. Diagnosis was based on the primary disease, e.g. children with UTIs and background nephrotic syndrome were classified as nephrotic syndrome, and those with AGN and renal failure were classified as AGN, not AKI. This was to avoid recruiting patients more than once simultaneously because of multiple diagnoses.

Hypertension was defined as blood pressure above the 95th percentile for the age and sex of the child. Proteinuria was determined using a urine dipstick and was not quantified. Some children with nephrotic syndrome were diagnosed before initiation of the study period and were already on steroids, their proteinuria had reduced to + or ++ . Nephrotic syndrome was diagnosed based on proteinuria, serum protein level, and cholesterol, in addition to clinical features.

UTI was diagnosed as presumptive, and was confirmed on receipt of urine culture results. Antenatal screening for congenital malformations was not captured as the children were post neonatal. Outcome measures were: discharged due to recovery of renal 
function; significant improvement but not full renal recovery discharged against medical advice; or death. Patients were followed up as outpatients at the nephrology clinic for variable periods.

Ethical approval was obtained from the hospital ethics committee.

Data entry and analysis were performed using Statistical Package for Social Science (SPSS) version 17 (SPSS Inc., USA).

\section{Results}

A total of 2658 children were admitted during the 30-month study period. Eighty-four (3.2\%) of the children were managed for various renal diseases; however, only 70 folders were utilised for the study as some had incomplete data, while others were missing.

There were 38 (54\%) males and $32(46 \%)$ females, giving a male: female ratio of 1.19:1. The mean (standard deviation (SD)) age was 88 (44) months, with a range of 1 - 168 months. Most children presented with a history of fever and reduction in urine volume (Table 1). On examination, most of the children were febrile and had facial swelling (Table 2).

Proteinuria was seen in most of the children (Table 3), while blood was not seen in the urine samples of most cases (Table 4). However, microscopy revealed that only $14(20 \%)$ had red blood cells in their urine, while 35 (50\%) had no red blood cells. Microscopy results were unavailable for $21(30 \%)$ children.

The diagnoses included UTI, had posterior urethral valve disorder (PUV), AGN, AKI with varying causes (mainly diarrhoea, sepsis and malaria), chronic kidney disease (CKD) with varying causes (chronic glomerulonephritis, nephrotic syndrome and others), and schistosomiasis (presented with severe anaemia) (Table 5). PUV disorder was the only congenital anomaly observed and no malignancy was identified in any of the cases. Renal biopsy was not performed in eligible children owing to a lack of funds.

\begin{tabular}{ll} 
Table $\mathbf{1 .}$ Symptoms of renal disease on presentation & $(\boldsymbol{N}=\mathbf{7 0})$ \\
\hline Symptom & $\boldsymbol{n}(\%)$ \\
\hline Fever & $44(63)$ \\
Reduction in urine volume/frequency & $32(46)$ \\
Body swelling & $30(43)$ \\
Abdominal pain & $28(40)$ \\
Vomiting & $26(37)$ \\
Dysuria & $19(27)$ \\
Macroscopic haematuria & $7(10)$ \\
Headache & $6(9)$ \\
Body rashes & $3(4)$ \\
Convulsion & $1(1)$ \\
*Some children presented with multiple symptoms. &
\end{tabular}

Table 2. Clinical features on presentation $(N=70)$

\begin{tabular}{ll}
\hline Sign ${ }^{*}$ & $n(\%)$ \\
\hline Febrile & $29(41)$ \\
Facial swelling & $28(40)$ \\
Pedal oedema & $27(39)$ \\
Ascites & $24(34)$ \\
Hypertension & $23(33)$ \\
Renal angle tenderness & $17(24)$ \\
Features of heart failure & $12(17)$ \\
*Some children presented with multiple signs. &
\end{tabular}

Fifty-nine (84\%) children were discharged, 47 (67\%) had normal renal function, and $12(17 \%)$ had residual renal function, i.e. were still on dialysis or still had deranged urea or creatinine. Seven (10\%) children died, while $4(6 \%)$ children's caregivers signed against medical advice and left the hospital. The children that died included 4 (57\%) with AGN, 2 (29\%) with AKI, and 1(14\%) with CKD, of which 4 (57\%) were males and 3 (43\%) were females.

\section{Discussion}

This study was the first in Gusau, Zamfara State, North-Western Nigeria to determine the prevalence, presentation, pattern and outcomes of childhood renal diseases. Studies from different parts of Nigeria and the world have reported variable patterns of renal diseases in children. Though our prevalence of $3.2 \%$ was low and may not be significant, it was similar to the $3.2 \%$ obtained in Calabar, ${ }^{[5]}$ $3.9 \%$ in Lagos, ${ }^{[6]} 3.0 \%$ in Libya, ${ }^{[7]}$ and $3.3 \%$ in Pakistan. ${ }^{[8]}$ However, it was lower than the $4.0 \%$ reported by Okoro et al. ${ }^{[9]}$ in Enugu and $6.3 \%$ by Bhatta et al. ${ }^{[10]}$ in Nepal. These variations could be related to methodology, especially with respect to the type and duration of the study, sample size, and variations in the rates of the renal disorders.

More males presented than females, which was similar to results obtained in Lagos, ${ }^{[6]}$ Libya, ${ }^{[7]}$ Enugu, ${ }^{[9]}$ and Jordan, ${ }^{[11]}$ although the differences were marginal. Complaints on presentation were similar to what was obtained in Sudan, ${ }^{[12]}$ though with varying frequencies.

Table 3. Proteinuria dipstick results $(N=70)$

\begin{tabular}{ll}
\hline Result & $\boldsymbol{n}(\%)$ \\
\hline+ & $23(33)$ \\
++ & $12(17)$ \\
+++ & $3(4)$ \\
++++ & 0 \\
Negative & $24(34)$ \\
No result & $8(11)$
\end{tabular}

Table 4. Presence of blood on dipstick $(N=70)$

\begin{tabular}{ll}
\hline Result & $n(\%)$ \\
\hline+ & $10(14)$ \\
++ & $13(19)$ \\
+++ & $3(4)$ \\
++++ & $2(3)$ \\
Negative & $34(49)$ \\
No result & $8(11)$
\end{tabular}

Table 5. Diagnosis of renal diseases $(N=70)$

\begin{tabular}{ll}
\hline Diagnosis & $\boldsymbol{n}(\%)$ \\
\hline UTI & $24(35)$ \\
AGN & $17(24)$ \\
AKI & $14(20)$ \\
Nephrotic syndrome & $8(11)$ \\
CKD & $6(9)$ \\
Schistosomiasis & $1(1)$ \\
UTI = urinary tract infection; AGN = acute glomerulonephritis; AKI = acute kidney \\
injury; CKD = chronic kidney disease.
\end{tabular}


The reason for the similarity may be because UTI was the most common diagnosis in the Sudanese study.

The childhood renal diseases identified in our study were similar to what was obtained in studies conducted elsewhere in Nigeria ${ }^{[1,2,5,6,13,14]}$ and the world; ; ${ }^{[7,15-17]}$ however, with varying rates. This observation could be explained by the fact that environmental factors, such as poor hygiene, poverty, and socioeconomic conditions, as well as genetic factors and late presentation, may have influenced the rates of childhood kidney diseases in our setting.

In our study, UTI was the most common renal disorder, which was similar to the findings of studies conducted in Pakistan, ${ }^{[8]}$ Sudan, ${ }^{[12]}$ Benin,${ }^{[13]}$ Port Harcourt, ${ }^{[14]}$ and Venezuela, ${ }^{[16]}$ but differed from findings in Jos, ${ }^{[2]}$ Calabar,${ }^{[5]}$ Lagos, ${ }^{[6]}$ Enugu, ${ }^{[9]}$ and Iran.${ }^{[15]}$ This was followed by AGN and AKI, which showed that infectious agents play a role in renal diseases in Gusau, as seen in other reports..$^{[9,13,14]}$ Congenital renal anomalies and malignancies were uncommon findings.

Most of our patients were discharged with good renal function, which was similar to the outcomes of the studies conducted in Enugu $^{[9]}$ and Sudan. ${ }^{[12]}$ Considering the fact that our study was conducted in a resource-poor setting with inadequate facilities for paediatric dialysis, the mortality rate of $10 \%$ was relatively low and similar to the $6.8 \%$ observed in Sudan, ${ }^{[12]}$ although higher than the $<1.0 \%$ reported in Libya, ${ }^{[7]}$ and significantly lower than the $17.7 \%$ reported in Lagos. Mortality was highest among children with AGN, similar to reports from Lagos. ${ }^{[6]}$ The reason for the mortality can be explained by the fact that most of the children had AKI and, in our setting, some patients present late to the hospital. All the deaths recorded in our study were due to AGN, AKI, or CKD. This observation may be attributed to a lack of dialysis for younger children, as peritoneal dialysis is not available in Zamfara State, late presentation, and cost of haemodialysis, all of which were common findings in various studies. ${ }^{[1,9,11]}$

\section{Study limitations}

This was a retrospective study, and therefore there may be inaccuracies in the data. Owing to a lack of adequate and sophisticated facilities, many renal disease cases may have been missed and poor socioeconomic conditions, along with the high cost of investigations, meant that investigations could not be performed for some of the children. A lack of peritoneal dialysis and the high cost of haemodialysis affected our mortality rate. Indeed, with these limitations, the true incidence of renal diseases may be underestimated. Our study can be improved upon by conducting a prospective study and obtaining grants for research to include appropriate investigations in the future.

\section{Conclusion}

Our study highlighted that the prevalence of childhood renal diseases in Gusau, Zamfara State, Nigeria was low, with males predominating. Fever, reduction in urine volume and frequency, body swelling, and abdominal pain were the most common presenting symptoms. UTI was the most common cause of renal disease requiring hospitalisation, followed by AGN. Congenital anomalies and malignancies were rare. Most of the children were discharged with normal renal function and the mortality was highest in children with AGN.

These data could be utilised by researchers and stakeholders in a resource-poor setting, such as ours, to plan for preventive nephrology, as UTI, AGN and AKI are largely preventable.

Acknowledgements. None.

Author contributions. GBI conceptualised and designed the study, and analysed and interpreted the data. All authors drafted the manuscript and approved the final version.

Funding. None.

Conflicts of interest. None.

1. Abdurrahman MB, Babaoye FA, Aikhionbare HA. Childhood renal disorders in Nigeria. Pediatr Nephrol 1990;4(1):88-93. https://doi.org/10.1007/bf00858449

2. Ocheke IE, Okolo SN, Bode-Thomas F, Agaba EI. Pattern of childhood renal disease in Jos, Nigeria: A preliminary report. J Med Trop 2010;12(2):52-55. https://doi.org/10.4314/jmt.v12i2.69316

3. Adedoyin OT, Adesiyun OA, Mark F, Adeniyi A. Childhood renal disorders in Ilorin, North Central Nigeria. Niger Postgraduate Med J 2012:19(2):88-91.

4. Bhimma R, Kalo U. Childhood kidney diseases in developing countries: Is it a forgotten disease? S Afr J Child Health 2016;10(2):103-104. https://doi. org/10.7196/SAJCH.2016.v1012.1144

5. Etuk IS, Anah MU, Ochighs SO, Eyong M. Pattern of paediatric renal disease in inpatients in Calabar, Nigeria. Trop Doct 2006;36(4):256. https://doi. org/10.1258/004947506778604968

6. Onifade EU. A ten year review of childhood renal admissions into the Lagos University Teaching Hospital, Nigeria. Nig Q J Hosp Med 2003;13(3-4):1-5. https:// doi.org/10.4314/nqihm.v13i3-4.12644

7. Elzouki AY, Amin F, Jaiswa OP. Prevalence and pattern of renal diseases in Eastern Libya. Arch Dis Child 1983;58(2):106-109. https://doi.org/10.1136/ adc.58.2.106

8. Iqbal J, Rahman MA, Khan MA. Pattern of renal diseases in children. J Pak Med Assoc 1994;44(5):118-120.

9. Okoro BA Okafor HU. Pattern of childhood renal disorders in Enugu. Niger J Paed 1999;26(1):14-18.

10. Bhatta NK, Shrestha P, Budhathoki S, et al. Profile of renal diseases in Nepalese children. Kathmandu Uni Med J 2008;6(2):191-194.

11. Hazza I, Mughraby H, Najada A. Spectrum of paediatric renal diseases in Jordan. Saudi J Kidney Dis Transpl 1997;8(3):314-316. http://www.sjkdt.org/ text.asp?1997/8/3/314/39362

12. Ali EM, Abdurrahman AH, Karrar ZA. Pattern of outcome of renal diseases in hospitalised children in Khartoum State, Sudan. Sudan J Paediatr 2012;12(2):52-59.

13. Ibadin OM, Ofovwe EG. Pattern of renal disease in children in mid western zone of Nigeria. Saudi J Kidney Transpl 2003;14(4):539-544.

14. Eke FU, Eke NN. Renal disorders in children: a Nigerian study. Pediatr Nephrol 1994;8(3):383-386. https://doi.org/10.1007/bf00866371

15. Derakhshan A, Al Hashemi GH, Fallahzadeh MH. Spectrum of in-patient renal diseases in children: A report from southern part of Islamic Republic of Iran. Saudi J Kidney Dis Transpl 2004;15(1):12-17.

16. Orta-Sibu N, Lopez M, Moriyon JC, Chavez JB. Renal diseases in children in Venezuela, South America. Pediatr Nephrol 2002;17(7):566-569. https://doi. org/10.1007/s00467-002-0892-4

17. Abdurrahman MB, Elidrissy ATH. Childhood renal disorders in Saudi Arabia. Paediatr Nephrol 1988;2(3):368-372. https://doi.org/10.1007/bf00858694 